\title{
NOTE ON CONGRUENCE CLASSES OF $n$-GROUPS
}

\author{
Janez Ušan
}

\begin{abstract}
In the paper the following proposition is proved. Let $(Q, A)$ be an $n$-group, $|Q| \in N \backslash\{1\}$, and let $n \geq 3$. Further on, let $\Theta$ be an arbitrary congruence of the $n-\operatorname{group}(Q, A)$ and let $C_{t}$ be an arbitrary class from the set $Q / \Theta$. Then there is a $k \in N$ such that the pair $\left(C_{t}, \stackrel{k}{A}\right)$ is a $(k(n-1)+1)$-subgroup of the $(k(n-1)+1)$-group $(Q, \stackrel{k}{A})$.
\end{abstract}

\section{Preliminaries}

1.1. Definition: Let $(Q, A)$ be an $n$-groupoid and $n \geq 2$. We say that $(Q, A)$ is a Dörnte $n$-group [briefly: $n$-group] iff is $n$-semigroup and an $n$-quasigroup as well.

1.2. Proposition [8]: Let $(Q, A)$ be an $n$-groupoid and $n \geq 2$. Then the following statements are equaivalent: $(i)(Q, A)$ is an $n$-group; (ii) there are mappings ${ }^{-1}$ and $\mathbf{e}$, respectively, of the sets $Q^{n-1}$ and $Q^{n-2}$ into the set $Q$ such that the following laws hold in the algebra $\left(Q,\left\{A,,^{-1}, \mathbf{e}\right\}\right)$ lof the type $<n, n-1, n-2>$ ]

(a) $A\left(x_{1}^{n-2}, A\left(x_{n-1}^{2 n-2}\right), x_{2 n-1}\right)=A\left(x_{1}^{n-1}, A\left(x_{n}^{2 n-1}\right)\right)$,

(b) $A\left(\mathbf{e}\left(a_{1}^{n-2}\right), a_{1}^{n-2}, x\right)=x$ and

(c) $A\left(\left(a_{1}^{n-2}, a\right)^{-1}, a_{1}^{n-2}, a\right)=\mathbf{e}\left(a_{1}^{n-2}\right)$; and

(iii) there are mappings ${ }^{-1}$ and $\mathbf{e}$, respectively, of the sets $Q^{n-1}$ and $Q^{n-2}$ into the set $Q$ such that the following laws hold in the algebra $(Q,\{\cdot, \varphi, b\})$ [of the type $<n, n-1, n-2>$ ]

(a) $A\left(A\left(x_{1}^{n}\right), x_{n+1}^{2 n-1}\right)=A\left(x_{1}, A\left(x_{2}^{n+1}\right), x_{n+2}^{2 n-1}\right)$,

(b) $A\left(x, a_{1}^{n-2}, \mathbf{e}\left(a_{1}^{n-2}\right)\right)=x$ and

( $\bar{c}) A\left(a, a_{1}^{n-2},\left(a_{1}^{n-2}, a\right)^{-1}\right)=\mathbf{e}\left(a_{1}^{n-2}\right)$.

Key words and phrases: $n$-semigroup, $n$-quasigroup, $n$-group. 
1.3. Remark: e is an $\{1, n\}$-neutral operation of $n$-groupoid $(Q, A)$ iff algebra $(Q,\{A, \mathbf{e}\})$ of the type $<n, n-2>$ satisfies the laws $(b)$ and $(\bar{b})$ from 1.2[4].

1.4. Proposition (Hosszú-Gluskin Theorem) [2, 3]: For every $n$-group $(Q, A), n \geq 3$, there is an algebra $(Q,\{\cdot, \varphi, b\})$ such that the following statements hold: $1^{\circ}(Q, \cdot)$ is a group; $2^{\circ} \varphi \in \operatorname{Aut}(Q, \cdot) ; 3^{\circ} \varphi(b)=b$; for every $x \in Q, \varphi^{n-1}(x) \cdot b=b \cdot x ;$ and $5^{\circ}$ for every $x_{1}^{n} \in Q, A\left(x_{1}^{n}\right)=$ $x_{1} \cdot \varphi\left(x_{2}\right) \cdots \cdot \varphi^{n-1}\left(x_{n}\right) \cdot b$.

1.5. Definition [5]: We say that an algebra $(Q,\{\cdot, \varphi, b\})$ is a HosszúGluskin algebra of order $n(n \geq 3)$ [briefly: $n H G$-algebra] iff $1^{\circ}-4^{\circ}$ from 1.4 hold. In adittion, we say that an $n H G$-algebra $(Q,\{\cdot, \varphi, b\})$ is associated to the $n$-group $(Q, A)$ iff $5^{\circ}$ from 1.4 holds.

\section{Auxiliarly proposition}

2.1. Proposition [5]: Let $(Q, A)$ be an $n$-group, e its $\{1, n\}$-neutral operatopn [:1.3] and $n \geq 3$. Further on, let $c_{1}^{n-2}$ be an arbitrary sequence over $Q$ and let for every $x, y \in Q$

(1) $B_{\left(c_{1}^{n-2}\right)}(x) \stackrel{\text { def }}{=} A\left(x, c_{1}^{n-2}, y\right)$,

(2) $\varphi_{\left(c_{1}^{n-2}\right)}(x, y) \stackrel{\text { def }}{=} A\left(\mathbf{e}\left(c_{1}^{n-2}\right), x, c_{1}^{n-2}\right)$ and

(3) $b_{\left(c_{1}^{n-2}\right)} \stackrel{\text { def }}{=} A\left(\overline{\mathbf{e}\left(c_{1}^{n-2}\right)}\right)$.

Then, the following statements hold:

(4) $\left(Q,\left\{B_{\left(c_{1}^{n-2}\right)}, \varphi_{\left.c_{1}^{n-2}\right)}, b_{\left(c_{1}^{n-2}\right)}\right\}\right)$ is an $n H G$-algebra associated to the $n$-group $(Q, A)$; and

(5) $\mathcal{C}_{A} \stackrel{\text { def }}{=}\left\{\left(Q,\left\{B_{\left(c_{1}^{n-2}\right)}, \varphi_{\left(c_{1}^{n-2}\right)}, b_{\left(c_{1}^{n-2}\right)}\right\}\right) \mid c_{1}^{n-2} \in Q\right\}$ is the set of all $n H G$-algebras associated to the $n$-group $(Q, A)$.

2.2. Proposition [10]: Let $(Q, A)$ be an $n$-group and let $n \geq 3$. Further on, let $\Theta$ be an arbitrary congruence of the $n-$ group $(Q, A)$. Then, for every $C_{t} \in Q / \Theta$ there is an $n H G$-algebra $(Q,\{\cdot, \varphi, b\})$ associated to the $n$-group $(Q, A)$ such that the following statements hold:

(i) $\left(C_{t}, \cdot\right) \triangleleft(Q, \cdot)$;

(ii) $\left(C_{t}, \varphi\right)$ is a 1-quasigroup; and

(iii) $\left(C_{t}, A\right)$ is an $n$-subgroup of the $n$-group $(Q, A)$ iff $b \in C_{t}$.

2.3. Remark: Let $\mathbf{e}$ be a $\{1, n\}$-neutral operation of the $n$-group $(Q, A)$ [:1.3]. Then: a) for $n=3$, e $\in Q$ !; and b) for $n>3,(Q, \mathbf{e})$ is an 
(n-2)-quasigroup [5]. $n H G$-algebra in Prop. 2.2. is defined with $\mathbf{e}\left(c_{1}^{n-2}\right)=t$ and with (1) - (3) from Prop. 2.1.

2.4. Proposition: Let $(Q, A)$ be an $n$-semigroup, $n \geq 2$ and let $(i, j) \in$ $N^{2}$. Let also $\stackrel{1}{A} \stackrel{\text { def }}{=} A$ and for every $m \in N$ and for every $x_{1}^{(m+1)(n-1)+1} \in Q$

$$
\stackrel{m+1}{A}\left(x_{1}^{(m+1)(n-1)+1}\right) \stackrel{\text { def }}{=} A\left(\stackrel{m}{A}\left(x_{1}^{m(n-1)+1}\right), x_{m(n-1)+2}^{(m+1)(n-1)+1}\right) .
$$

Then, for every $x_{1}^{(m+1)(n-1)+1} \in Q$ and for every $t \in\{1, \ldots, i(n-1)+1\}$, the following equality holds

$$
\stackrel{i}{A}\left(x_{1}^{t-1}, \stackrel{j}{A}\left(x_{t}^{t+j(n-1)}\right), x_{t+j(n-1)+1}^{(i+j)(n-1)+1}\right)=\stackrel{i+j}{A}\left(x_{1}^{(i+j)(n-1)+1}\right) .
$$

By 2.4 an by 1.1, we conclude that the following proposition holds:

2.5. Proposition: Let $(Q, A)$ be an $n-$ group, $n \geq 2$ and $i \in N$. Then $(Q, \stackrel{i}{A})$ is an $(i(n-1)+1)$-group.

2.6. Proposition [11]: Let $(Q, A)$ be an $n-$ group and $n \geq 2$. Then for every $k \in N \backslash\{1\}$ the following equality holds $\operatorname{Con}(Q, A)=\operatorname{Con}(Q, \stackrel{k}{A})$.

\section{Result}

3.1. Theorem: Let $(Q, A)$ be an $n$-group, $|Q| \in N \backslash\{1\}$ and let $n \geq 3$. Further on, let $\Theta$ be an arbitrary congruence of the $n$-group $(Q, A)[\Theta \in$ $\operatorname{Con}(Q, A)]$ and let $C_{t}[t \in Q]$ be an arbitrary class from the set $Q / \Theta$. Then there is a $k \in N$ such that the pair $\left(C_{t}, \stackrel{k}{A}\right)$ is a $(k(n-1)+1)$ - subgroup of the $(k(n-1)+1)-$ group $(Q, \stackrel{k}{A})$.

Proof. Firstly, we prove that under the assumptions the following statements hold:

$\overline{1}$ If $(Q,\{\cdot, \varphi, b\})$ is an $n H G$-algebra [:1.5], then for every $k \in N$ $\left(Q,\left\{\cdot, \varphi, b^{k}\right\}\right)$ is a $(k(n-1)+1) H G$-algebra; and

$\overline{2}$ If $(Q,\{\cdot, \varphi, b\})$ is an $n H G$-algebra associated to the $n$-group $(Q, A)$, then for every $k \in N\left(Q,\left\{\cdot, \varphi, b^{k}\right\}\right)$ is a $(k(n-1)+1) H G$-algebra associated to the $(k(n-1)+1)-\operatorname{group}(Q, A)^{1)}$.

The sketch of the proof of $\overline{1}$ :

a) $\varphi\left(b^{1}\right)=b, \varphi\left(b^{t}\right)=b^{t}$,

$$
\varphi\left(b^{t+1}\right)=\varphi\left(b^{t}\right) \cdot \varphi(b)=b^{t} \cdot b=b^{t+1}
$$

\footnotetext{
${ }^{1)}$ See 2.5 .
} 
b) $\varphi^{t(n-1)}(x) \cdot b^{t}=b^{t} \cdot x$

$$
\begin{aligned}
\varphi^{(t+1)(n-1)}(x) \cdot b^{t+1} & =\varphi^{t(n-1)}\left(\varphi^{n-1}(x)\right) \cdot b^{t} \cdot b \\
& =b^{t} \cdot \varphi^{n-1}(x) \cdot b \\
& =b^{t} \cdot b \cdot x \\
& =b^{t+1} \cdot x \quad[: 1.5] .
\end{aligned}
$$

The sketch of the proof of $\overline{2}$ :

a) $\stackrel{1}{A} \stackrel{2 \cdot 4}{=} A, A\left(x_{1}^{n}\right) \stackrel{1 \cdot 5}{=} x_{1} \cdot \varphi\left(x_{2}\right) \cdots \cdot \varphi^{n-1}\left(x_{n}\right) \cdot b$;

$\bar{b}) \stackrel{t}{A}\left(x_{1}^{t(n-1)+1}\right)=x_{1} \cdot \varphi\left(x_{2}\right) \cdots \cdot \varphi^{t(n-1)}\left(x_{t(n-1)+1}\right) \cdot b^{t}$;

c) $\stackrel{t+1}{A}\left(x_{1}^{(t+1)(n-1)+1}\right) \stackrel{2 \cdot 4}{=} A\left(x_{1}^{n-1}, \stackrel{t}{A}\left(x_{n}^{(t+1)(n-1)+1}\right)\right)=$ $x_{1} \cdot \varphi\left(x_{2}\right) \cdots \cdot \varphi^{n-2}\left(x_{n-1}\right) \cdot \varphi^{n-1} \stackrel{t}{A}\left(x_{n}^{(t+1)(n-1)+1}\right) \cdot b \stackrel{\bar{b})}{=}$ $x_{1} \cdot \varphi\left(x_{2}\right) \cdots \cdots \varphi^{n-2}\left(x_{n-1}\right) \cdot \varphi^{n-1}\left(x_{n}\right) \cdots \cdots \varphi^{(t+1)(n-1)}\left(x_{(t+1)(n-1)+1}\right) \cdot b^{t} \cdot b=$ $x_{1} \cdot \varphi\left(x_{2}\right) \cdots \cdots \varphi^{n-2}\left(x_{n-1}\right) \cdot \varphi^{n-1}\left(x_{n}\right) \cdots \cdots \varphi^{(t+1)(n-1)}\left(x_{(t+1)(n-1)+1}\right) \cdot b^{t+1}$.

Finally, by Proposition 2.6, by $\overline{1}, \overline{2}$, by $|Q| \in N \backslash\{1\}$, and by Proposition 2.2 , we conclude that the Theorem holds.

\section{Remark}

If $n \geq 3$, then: 1$)$ there exist $n$-group $(Q, A)$ and its congruence $\Theta$ such that for every $C_{a} \in Q / \Theta$ the pair $\left(C_{a}, A\right)$ is not an $n$-group [6,9]; 2) there exist an $n$-group $(Q, A)$ and its congruence $\Theta$ such that for every $C_{a} \in Q / \Theta$ the pair $\left(C_{a}, A\right)$ is an $n$-group $[6,7,9]$; and 3$)$ there exist $n$-group $(Q, A)$ and its congruence $\Theta$ such that exactly one $C_{a} \in Q / \Theta$ the pair $\left(C_{a}, A\right)$ is an $n$-group [6].

\section{References}

[1] W. D. Dörnte: Untersuchengen über einen verallgemeinerten Gruppenbegriff, Math. Z. 29(1928), 1-19.

[2] M. Hosszú: On the explicit form of $n$-group operations, Publ. Math. (Debrecen) 10(1963), 88-92.

[3] L. M. Gluskin: Positional operatives, (Russian), 68(110)(1965), 444-472.

[4] J. Ušan: Neutral operations of n-groupoids, (Russian), Rev. of Research, Fac. of Sci. Univ. of Novi Sad, Math. Ser. 18.2(1988), 117-126.

[5] J. Ušan: On Hosszú-Gluskin algebras corresponding to the same n-group, Rev. of Research, Fac. of Sci. Univ. of Novi Sad, Math. Ser. 25.1(1995), 101-119.

[6] B. Janeva: Congruences on n-groups, Mat. Bilten (Skopje) 45(1995), 85-90. 
[7] J. Ušan: On congruences on n-groups, Novi Sad J. Math. 27.2(1997), 89-100.

[8] J. Ušan: n-groups as variety of type $\langle n, n-1, n-2\rangle$, in: Algebra and Model Theory, (A. G. Pinus and K.N.Ponomaryov, eds.) Novosibirsk 1997, 182-208.

[9] J. Ušan: On congruence classes of n-groups, Novi Sad J. Math. 28.1(1998), 75-89.

[10] J. Ušan: Congruences of n-group and of associated Hosszú-Gluskin algebras, Novi Sad J. Math. 28.2.(1998), 91-108.

[11] J. Ušan: A comment on a class of polyadic group, Scientific Bulletin of the Polytechnic University Timişoara, Math.-Phys. Ser. 44(58)-2(1999), 8-14.

Institute of Mathematics University of Novi Sad

Trg D. Obradovića 4, 21000 Novi Sad, Yugoslavia 